Bryn Mawr College

Scholarship, Research, and Creative Work at Bryn Mawr

College

Physics Faculty Research and Scholarship

Physics

1999

\title{
Population Trapping in Extremely Highly Excited States in Microwave Ionization
}

\author{
Michael W. Noel \\ Bryn Mawr College, mnoel@brynmawr.edu \\ W.M. Griffith \\ T. F. Gallagher
}

Let us know how access to this document benefits you.

Follow this and additional works at: http://repository.brynmawr.edu/physics_pubs

Part of the Physics Commons

\section{Custom Citation}

Michael W. Noel, W. M. Griffith, and T. F. Gallagher, "Population Trapping in Extremely Highly Excited States in Microwave Ionization," Phys. Rev. Lett. 83, 1747 (1999).

This paper is posted at Scholarship, Research, and Creative Work at Bryn Mawr College. http://repository.brynmawr.edu/physics_pubs/76

For more information, please contact repository@brynmawr.edu. 


\title{
Population Trapping in Extremely Highly Excited States in Microwave Ionization
}

\author{
Michael W. Noel, W.M. Griffith, and T.F. Gallagher \\ Department of Physics, University of Virginia, Charlottesville, Virginia 22901
}

(Received 18 December 1998)

\begin{abstract}
When a lithium atom in a Rydberg state $(n \sim 80)$ is exposed to a short, intense microwave pulse we find that substantial population is left in extremely highly excited states $(n>120)$, in spite of the fact that the microwave field amplitude is more than 40 times larger than required to classically ionize these states.

PACS numbers: $32.80 . \mathrm{Rm}, 42.50 . \mathrm{Hz}$
\end{abstract}

The interaction between an atom and a strong oscillating field has revealed a multitude of interesting phenomena, often involving the ionization of the atom [1]. Among the most fascinating are those cases in which ionization does not occur, in spite of its appearing to be very probable at first glance. Several immediately relevant examples occur in the microwave ionization of Rydberg atoms. Classical stabilization of Rydberg atoms against microwave ionization occurs when the microwave frequency or one of its harmonics matches the classical Kepler frequency of the Rydberg atom [2-4]. Of particular interest for this work, it was observed long ago in classical calculations that the Rydberg atom could be left in very high lying states by a microwave pulse [5-7]. More recently there have been extensive calculations of the final state distributions subsequent to a microwave pulse [8-11]. At low microwave fields the final state population is found to fall off roughly exponentially with the change in $n$ of the final state from the initial state, and experiments have confirmed these predictions $[8,9]$. Here $n$ is the principal quantum number. At fields high enough to produce ionization, the calculated distributions of final states are continuous from the initially populated state to the continuum, with structure due to resonant single photon transitions [11]. To date, however, no experiments have been carried out to test these predictions.

Here we report the analysis of the final state distribution of lithium Rydberg atoms exposed to intense, short microwave pulses. We find the rather surprising result that when $n \sim 80$ lithium atoms are exposed to $0.5 \mathrm{~ns}$ long microwave pulses $>35 \%$ of the population is transferred to states of $n>120$, with no apparent population in states of $n<120$, contrary to the calculations, in spite of the fact that the microwave field amplitude exceeds the classical ionization field of $n=120$ by a factor of 40 . As the pulse is made longer the number of $n>120$ atoms surviving decreases, but it is still $\sim 3 \%$ with a 10 ns pulse. Recent measurements of Rydberg atoms kicked by a series of unipolar pulses also show atoms left in very high $n$ states, again in disagreement with calculations [12]. Using the fact that there can be no transfer of energy from the field to the electron when it is far from the ion core and accounting for the state redistribution that occurs when the electron comes near the core we have developed a simple picture that reproduces the observed population transfer to what have been termed extremely highly excited states [5].

The general scheme for this experiment was to excite lithium atoms to a high $n$ Rydberg state, allow them to interact with a short microwave pulse, and measure the state distribution of the atoms that remained bound. Lithium atoms, from an effusive source, entered a piece of WR90 waveguide through a small hole in one of its sides. Three nanosecond dye laser pulses entered the waveguide through a hole on the opposite side and excited the atoms to a Rydberg state through the $2 s \rightarrow 2 p, 2 p \rightarrow 3 s$, and $3 s \rightarrow n p$ transitions. The final laser was polarized orthogonal to the microwave field so as to excite $|m|=1$ states where $m$ is the magnetic quantum number. With the laser bandwidth of $4 \mathrm{GHz}$, we could selectively excite Rydberg states beyond $n=100$.

The microwave pulse was formed by mixing the $11.5 \mathrm{GHz}$ continuous wave output of a sweep oscillator with a fast rising edge $(\sim 100 \mathrm{ps})$ voltage step in one mixer and a fast falling edge $(\sim 100 \mathrm{ps})$ voltage step in a second mixer. The relative delay between the steps could be precisely adjusted to give the desired pulse width. A subsequent mixing stage with a 1 to $100 \mathrm{~ns}$ pulse (depending on the pulse length set with the fast steps) served to further suppress the wings of this fast pulse. The microwave source was phase locked to the pulse envelope, which allowed us to monitor the microwave electric field, not just the field envelope. After two stages of preamplification we passed the pulse through a fast microwave switch which was gated open for a time slightly longer than the pulse. Finally, the pulse was amplified in a gated traveling wave tube amplifier and passed through a voltage controlled attenuator with which we could vary its power over $40 \mathrm{~dB}$. The pulse was coupled into and out of the short piece of waveguide containing the prepared Rydberg atoms after which it was attenuated and monitored with a sampling oscilloscope. We calibrated the power using a Hewlett Packard 432A power meter and estimate a microwave field uncertainty of $10 \%$.

After the microwave interaction a negative field ionization pulse was applied to a septum below the atoms in the waveguide. The field ionized electrons passed through a 
small hole in the top of the waveguide and were collected with a dual microchannel plate detector. By adjusting a bias voltage on the septum to minimize excitation of high $n s$ states we reduced the static electric field to less than $50 \mathrm{mV} / \mathrm{cm}$, which can ionize states with $n>280$.

With the laser tuned to the desired Rydberg state we repetitively scanned the microwave amplitude over many laser shots as we downloaded the time resolved electron signals recorded on a digital oscilloscope on each shot. The time resolved electron signal revealed the state distribution of the atoms which remained bound after the microwave interaction. Electrons left in high $n$ states were ionized early during the slowly rising field ionization pulse and arrived early in time, whereas those of lower $n$ required higher fields and consequently arrived later in time. We calibrated the arrival time versus $n$ by directly exciting known states, and we can distinguish states up to about $n=130$, above which the electrons all arrive at the detector at the same time. Electrons which were ionized directly by the microwave pulse dispersed before the arrival of the field ionization pulse, so they were not registered at the detector.

In Fig. 1 we show a typical set of data collected according to the above prescription for an initial state of $n=80$ interacting with a $0.5 \mathrm{~ns}(5$ cycle) pulse. The Kepler period is given by $2 \pi n^{3}$ in atomic units, and for $n=80$ it is $78 \mathrm{ps}$. The primary classical resonance, where the Kepler period is equal to the microwave period,

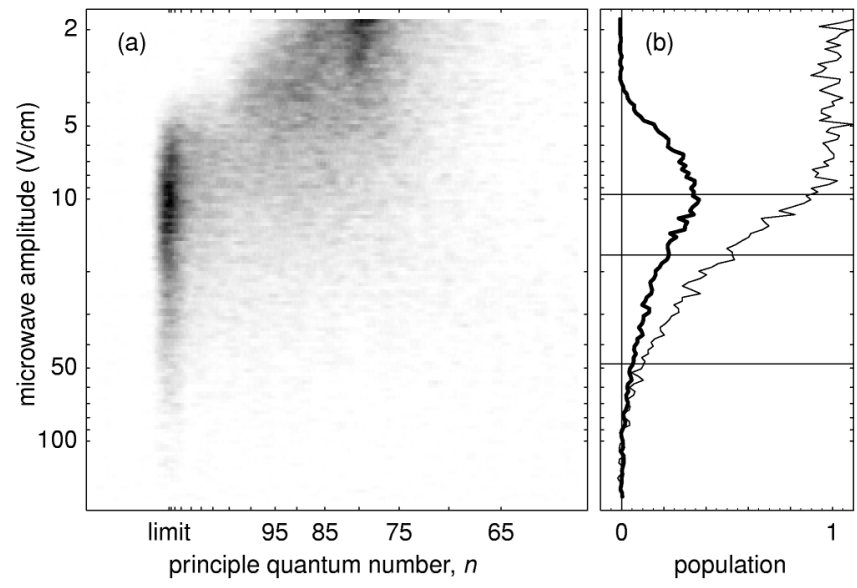

FIG. 1. Bound state distribution as a function of field strength for an atom initially prepared in the $80 p$ state interacting with a $0.5 \mathrm{~ns}$ pulse. In (a) electron signal is mapped to a gray scale with black corresponding to large signal and white to no signal. The horizontal axis, which experimentally is the detection time of an electron after being ionized by the slowly rising field ionization pulse, has been calibrated according to $n$. The labels are placed at the peak in the arrival time of the electron signal for a given $n$. Bound states above $n=130$ are not resolved and we label them collectively with "limit." (b) Electron signal integrated over all $n$ (light line) and electron signal integrated from $120<n<\infty$ (bold line). The three horizontal lines in (b) are the $10 \%, 50 \%$, and $90 \%$ ionization fields from top to bottom, respectively. occurs at $n=83$. The duration of the short $0.5 \mathrm{~ns}$ pulse matches the Kepler period at $n=150$. The state distribution as a function of field amplitude is plotted in Fig. 1(a) by mapping the electron signal to a gray scale with black corresponding to a large signal and white to no signal. Figure 1(b) shows the electron signal integrated over all $n$, the total remaining atom signal, as well as the integrated state population for $120<n<\infty$ as functions of field amplitude. Finally in Fig. 2 we show several lines out of the full data set in Fig. 1(a). These are the state distributions at microwave amplitudes of $2 \mathrm{~V} / \mathrm{cm}$ (no ionization), $9.5 \mathrm{~V} / \mathrm{cm}$ (10\% ionization), and $48 \mathrm{~V} / \mathrm{cm}$ (90\% ionization).

In both Figs. 1 and 2 it is apparent that when a significant fraction of the atoms is ionized, a surprisingly large fraction of the remaining bound population has been transferred to high $n$ states (as much as 35\%). Furthermore, some population remains bound in these high $n$ states for fields as high as $60 \mathrm{~V} / \mathrm{cm}$, which is more than 40 times the field necessary to classically ionize an atom of $n=120$.

In an effort to explain the remarkable accumulation of population in these high $n$ states, even in very strong microwave fields, we consider the interaction of a classical Rydberg atom with the microwave field. If the electron is far from the core it simply oscillates in the field as if it were a free electron. The amplitude of the oscillations for a $60 \mathrm{~V} / \mathrm{cm}$ field is $0.2 \mu \mathrm{m}$, much less than the size of the orbit for $n>120$. In contrast, when the electron returns to the core it can gain energy from or lose it to the oscillating field, depending on the phase of the field. Since the orbit time for $n>150$ is longer than our microwave pulse, if such states are formed in the first cycle of the pulse, the field is off by the time the electron returns to the core, and the electron remains bound in these high $n$ states.

With a $9.5 \mathrm{~ns}$ (110 cycle) pulse there is still some excitation of high $n$ states, however, the amount of population transferred to these states is much smaller. As shown by Fig. 3, the maximum transfer for an atom prepared in the $80 p$ state is about $3 \%$. In this case

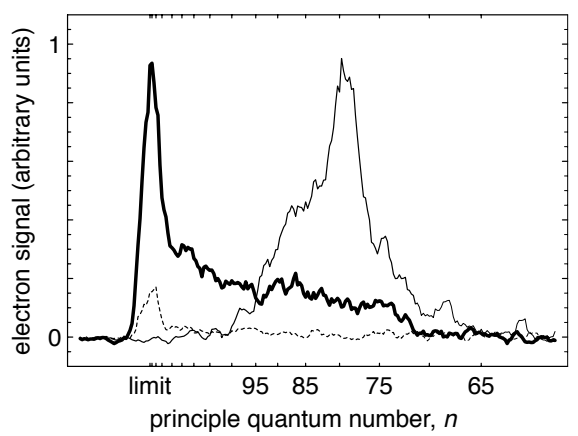

FIG. 2. State distributions from Fig. 1 for field amplitudes of $2 \mathrm{~V} / \mathrm{cm}$ (no ionization, light line), $9.5 \mathrm{~V} / \mathrm{cm}$ (10\% ionization, bold line), and $48 \mathrm{~V} / \mathrm{cm}$ (90\% ionization, dotted line). 


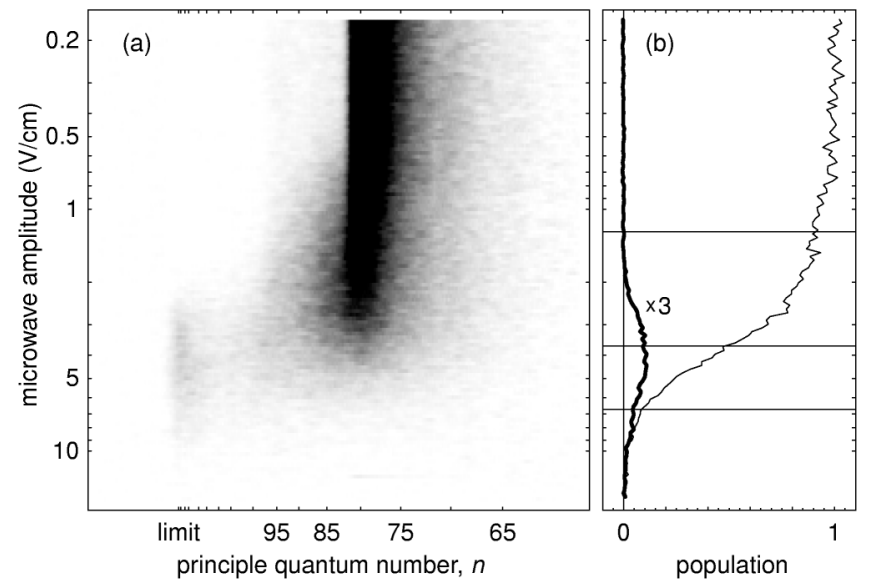

FIG. 3. (a) State distribution as a function of field strength for an atom initially prepared in the $80 p$ state interacting with a $9.5 \mathrm{~ns}$ pulse. (b) Electron signal integrated over all $n$ (light line) and electron signal integrated from $120<n<\infty$ (bold line). For clarity the bold line in (b) has been expanded by a factor of 3 .

the Kepler period does not exceed the pulse width until $n>400$, and a wave packet at $n=280$, the highest lying bound state possible in our experiment, passes the core 3 times during the microwave pulse. Each time the electron passes the core there is some probability that it will be ionized by the microwave field, so with 110 field cycles it is not surprising that few bound atoms are left. As might be expected, with a $100 \mathrm{~ns}(1150$ cycle $)$ pulse we did not see any excitation to high $n$ states for any of the initial states used ( $n=47$ to $n=94)$. In this case the Kepler period does not exceed the pulse width until $n>870$ and at $n=280$ the electron passed the core 30 times during the microwave pulse.

The dynamic nature of the above classical description can also be incorporated into a simple model for the final state distributions. In this model we consider the microwave interaction one cycle at a time in the following iterative procedure. The effect of the first cycle is to spread the population from its initial state over an energy band of states. The next cycle spreads the population of each of these states in an identical way for $n<n_{1}$ and does nothing for states with $n>n_{1}$, where $n_{1}$ is the principle quantum number at which the Kepler period is equal to the microwave period. On the third field cycle atoms put into states with $n_{1}<n<n_{2}$, by the first cycle, where $n_{2}$ is the principal quantum number for which the Kepler period is twice the microwave period, as well as atoms put into states with $n<n_{1}$ by the second field cycle, are spread over the energy band. This process is repeated for each field cycle, with those atoms that have returned to or remained near the ion core being spread over the energy band.

The evolution of the state distribution during this iteration process is shown in Fig. 4 for a five cycle pulse interacting with an atom initially in $n=80$. The function

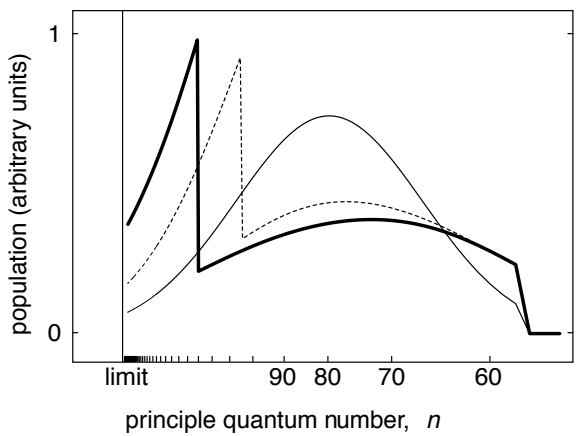

FIG. 4. Model of the state distributions for the interaction between a five cycle pulse and an $n=80$ Rydberg atom. We show distributions after the first (light line), third (dotted line), and fifth (bold line) cycles.

used to spread population was a Gaussian whose width was adjusted so that, after five iterations, $10 \%$ of the final distribution had positive energy for comparison to the $10 \%$ ionization trace in Fig. 2. For low $n$ states the microwave field oscillates slowly compared to the classical period, and ionization proceeds via field ionization rather than through the diffusion process seen when the time scales are comparable. In order to account for this effect the Gaussian was cut off sharply at $n=58$, where the microwave period is 3 times the Kepler period. This model contains three essential parameters, the width of the distribution with which we spread population and the low and high $n$ cutoffs for the interaction, all of which have clear physical origins. The gross features of the final distribution, the peak at high $n$, and the broad tail to lower $n$, are relatively insensitive to changes in these parameters of the model, and it is evident that the model captures the essential features of the data shown in Fig. 2.

Finally, we have studied the excitation to the high $n$ states as a function of the initial state populated. In Fig. 5(a) we show, using a gray scale, the population of high $n$ states $(120<n<\infty)$ as a function of field amplitude for the series of initial states from $n=50$ to $n=95$. Figure 5(b) is composed of lines out of Fig. 5(a) for selected initial states. In Fig. 5(a) we also plot the $10 \%$ microwave ionization fields for lithium, which exhibit a resonant structure similar to that seen in hydrogen [3]. It is evident that the excitation to very high $n$ states occurs only for fields sufficient to produce some ionization, which is not surprising since producing these high $n$ states is not so different from producing free electrons. In Fig. 5(a) we have marked the positions at which the microwave frequency is equal to or a subharmonic of the classical Kepler frequency $\left(1 / 2 \pi n^{3}\right.$ in atomic units). It is evident that the production of the very highly excited states is maximized near the fundamental resonance, but exactly why is not apparent.

In conclusion, when Rydberg atoms are exposed to intense microwave pulses a remarkable amount of population is left in states with $n>120$ in spite of the 

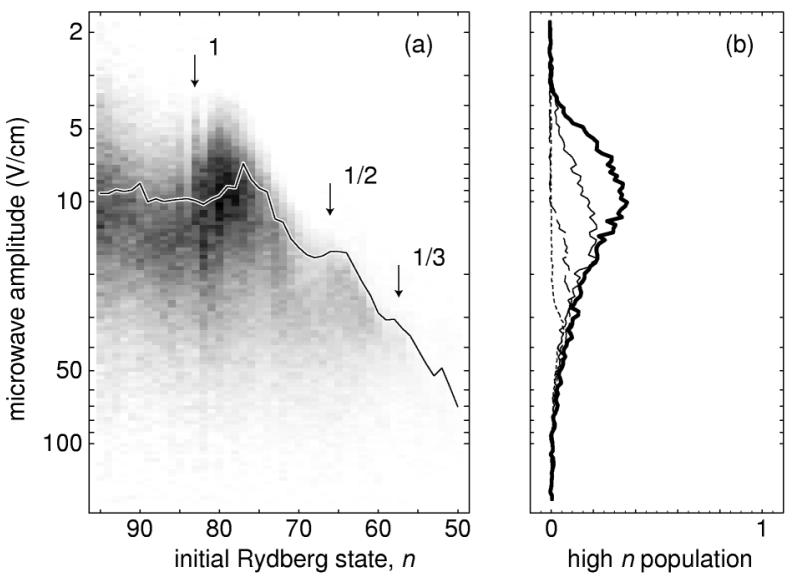

FIG. 5. Redistribution to states of $120<n<\infty$ from various initial states. In (a) we represent the high $n$ populations with a gray scale for initial states ranging from $n=50$ to $n=95$. The line in (a) is the $10 \%$ ionization field for the different initial states and the arrows mark the points at which the microwave frequency is equal to, one-half, and one-third the classical frequency. In (b) we show the population of high $n$ states as a function of field amplitude for initial states of $n=60$ (dotted line), $n=70$ (dashed line), $n=80$ (bold line), and $n=90$ (thin line).

fact that the microwave amplitude is 40 times larger than the classical ionization field for these states. The excitation to these weakly bound states has an onset matching the microwave ionization threshold field and is most pronounced for a microwave frequency near the Kepler frequency. Although unexpected, this behavior can be explained by a simple model based on the likelihood of the Rydberg electron's absorbing energy from the field at different points in a classical orbit.
This work was supported by the Air Force Office of Scientific Research and the National Science Foundation. We thank R.R. Jones and P.M. Koch for useful discussions.

[1] Atoms in Intense Laser Fields, Advances in Atomic, Molecular, and Optical Physics, edited by M. Gavrila (Academic, San Diego, 1992), Suppl. 1.

[2] K.A.H. van Leeuwen, G.V. Oppen, S. Renwick, J.B. Bowlin, P.M. Koch, R. V. Jensen, O. Rath, D. Richards, and J.G. Leopold, Phys. Rev. Lett. 55, 2231 (1985).

[3] P. M. Koch and K. A.H. van Leeuwen, Phys. Rep. 255, 289 (1995).

[4] M.M. Sanders and R.V. Jensen, Am. J. Phys. 64, 21 (1996).

[5] J. G. Leopold and I. C. Percival, Phys. Rev. Lett. 41, 944 (1978).

[6] J. G. Leopold and I. C. Percival, J. Phys. B 12, 709 (1979).

[7] D. A. Jones, J. G. Leopold, and I. C. Percival, J. Phys. B 13, 31 (1980).

[8] J. N. Bardsley, B. Sundaram, L. A. Pinnaduwage, and J. A. Bayfield, Phys. Rev. Lett. 56, 1007 (1986).

[9] R. Blumel, A. Buchleitner, R. Graham, L. Sirko, U. Smilansky, and H. Walther, Phys. Rev. A 44, 4521 (1991).

[10] G. Casati, I. Guarneri, and D. L. Shepelyansky, Phys. Rev. A 36, 3501 (1987).

[11] R. V. Jensen, S.M. Susskind, and M.M. Sanders, Phys. Rep. 201, 1 (1991).

[12] M.T. Frey, F.B. Dunning, C. O. Reinhold, S. Yoshida, and J. Burgdörfer, Phys. Rev. A 59, 1434 (1999). 